\title{
Assessment of antibacterial potential of methanol, n-hexane, ethyl acetate and chloroform Moringa oliefera leaf extracts
}

\author{
Nudrat Rafiq ${ }^{1}$, Anser Ali ${ }^{*}$, Huma Khurshid ${ }^{1}$, Bilal Akbar ${ }^{2}$, Zahid \\ Hassan Tarar ${ }^{3}$, Fahad Nazir ${ }^{4}$, Hamza Javed ${ }^{5}$, Iftikhar Ahmed ${ }^{6}$ and \\ Mushtaq Ahmed ${ }^{1}$ \\ 1. Department of Zoology, Mirpur University of Science and Technology (MUST), Mirpur-10250 (AJK)- \\ Pakistan \\ 2. Department of Mechanical Engineering, Mirpur University of Science and Technology (MUST), Mirpur- \\ 10250 (AJK)-Pakistan \\ 3. Soil and Water Testing Laboratory, Mandi Bahauddin-Pakistan \\ 4. Department of Entomology, Faculty of Crop Protection, Sindh Agriculture University Tandojam-Pakistan \\ 5. Department of Medical Laboratory Technology, Government College University Faisalabad-Pakistan \\ 6. National Agricultural Research Centre (NARC), Park Road, Islamabad-45500-Pakistan \\ *Corresponding author's email: anser.zoology@must.edu.pk \\ Citation \\ Nudrat Rafiq, Anser Ali, Huma Khurshid, Bilal Akbar, Zahid Hassan Tarar, Fahad Nazir, Hamza Javed, Iftikhar \\ Ahmed and Mushtaq Ahmed. Assessment of antibacterial potential of methanol, n-hexane, ethyl acetate and \\ chloroform Moringa oliefera leaf extracts. Pure and Applied Biology. Vol. 9, Issue 3, pp1946-1953. \\ http://dx.doi.org/10.19045/bspab.2020.90207
}

\begin{tabular}{llll}
\hline \hline Received: 20/02/2020 & Revised: 27/04/2020 & Accepted: 09/05/2020 & Online First: 29/05/2020 \\
\hline \hline
\end{tabular}

\section{Abstract}

Bacterial infections and their increasing resistance to common antibiotics is posing serious threat to global public health. To this end, finding new alternatives and evaluating their antibacterial efficacy is always desirable. Therefore, in the present study we used methanol $(\mathrm{MeOH}), \mathrm{n}$-hexane (n-Hex), ethyl acetate (ETAC) and chloroform $\left(\mathrm{CHCl}_{3}\right)$ to prepared four different types of extracts from Moringa oleifera ( $M$. oliefera) leaves aiming to inhibit five selected bacteria. Initially, agar well diffusion methods was used, and zones of inhibition were measured as an indicator of bacterial susceptibility. $\mathrm{MeOH}, \mathrm{n}-\mathrm{Hex}, \mathrm{ETAC}$ and $\mathrm{CHCl}_{3}$ leaf extracts showed highest zone of inhibition against E. coli, B. cereus, S. pyogenes, S. aureus and S. enterica, respectively. Moreover, highest zones of inhibition were observed at lowest incubation (24hr) and lowest zones were observed at highest incubation period (72hr) for all tested concentrations. Later, macrodilution method was used to access the antibacterial susceptibility in liquid medium. Results confirmed the susceptibility of all test bacteria with different level of $\mathrm{IC}_{50}$ values ranging from $7.07 \pm 0.44$ to $10.91 \pm 0.10 \mathrm{mg} / \mathrm{ml}$ for $\mathrm{MeOH}$ extract, $1.66 \pm 0.08$ to $2.11 \pm 0.11 \mathrm{mg} / \mathrm{ml}$ for $\mathrm{n}$-Hex extract, $2.58 \pm 0.13$ to $3.84 \pm 0.21$ $\mathrm{mg} / \mathrm{ml}$ for ETAC extract and $3.73 \pm 0.75$ to $8.36 \pm 0.20 \mathrm{mg} / \mathrm{ml}$ for $\mathrm{CHCl}_{3}$ extract. Interestingly, none of the tested bacteria showed resistance against any of the tested extract in well diffusion or macrodilution method expressing the $M$. oliefera leaves extracts as potent candidates to kill bacteria in semisolid or in liquid medium to fulfill medical needs in future.

Keywords: Bacteria; M. oleifera; Macrodilution method; Zones of inhibition

Introduction

Antibiotics have been frequently used

worldwide and saved countless lives.
However, it is evident that the success of antibiotics might only be temporary, and we now expect a long-term or perhaps never- 
ending challenge to find new effective alternatives to combat increasing problem of antibiotic resistance. The number of antibiotics such as trimethoprim, nitrofurantoin, fluoroquinolones, fosfomycin, gentamicin, sulfamethoxazole, cephalexin linezolid, telavancin daptomycin, vancomycin and clindamycin are easily available. However, bacterial resistance is reported to increase continuously at an alarming rate [1, 2]. For example, E. coli strains are found to be resistant to ampicillin, ciprofloxacin, cephalosporins, trimethoprimsulfamethoxazole [3], S. aureus strains are found to be resistant to ampicillin, penicillin [4] and, B. cereus strains are found to be resistant to cephalosporin, penicillin. Resistance to clindamycin, cefazolin, cefotaxime and trimethoprimsulfamethoxazole is also reported [5]. Moreover, S. enterica developed resistance to macrolides, aminoglycosides, cephalosporin, tetracycline, ketolides ampicillin [6] and $S$. pyogenes strain developed resistance to most macrolides, floroquinolone macrolide, lincosamide and streptogramin antibiotics [7]. Therefore, search for new effective alternatives to combat microbial infections is always desirable.

Medicinal plants have been proven a good source of antibacterial therapy and have been used to treat diseases all over the world for many decades. Based on pharmacopoeias and study of medical plants in 91 countries, World Health Organization (WHO) reported almost 20,000 medicinal plant species [8]. Plants that inhibit the growth of microorganisms are important for human health and have been studied since 1926 [9, 10]. Herbal medicines being safe and environment friendly gained increasing popularity and are widespread around the globe encouraging to explore their biological properties to unmet the medical needs.

Thus, in the present study Moringa oliefera, one of the best known and widely distributed specie of a monogeneric family Moringaceae [11] is selected for the evaluation of antibacterial properties. Selected plant ranges 5 to $10 \mathrm{~m}$ in height [12] and found wild near the sandy beds of rivers and streams or cultivated in plains, hedges and in-house yards. The numbers of medicinal properties have been associated to various parts of this highly esteemed tree. Plant parts including root, bark, leaf, fruit, gum, flowers and seed have been used for various ailments including infectious diseases, inflammation, gastrointestinal, cardiovascular, hematological and hepatorenal disorders [13, 14]. Moringa leaves are rich source of protein, vitamin $\mathrm{C}, \beta$-carotene, calcium and potassium and have flavonoids, phenolics, carotenoids and ascorbic acid which serve as a valuable natural antioxidant and good food preserver [15, 13]. Interestingly, its leaves have been used to treat headaches, piles, sore throat, bronchitis, fevers, eye and ear infections, scurvy, diabetics as well as glandular swelling [12]. The juice from its root bark is used to put into ears to relieve earaches, placed in a tooth cavity as a pain killer and, has shown promising antitubercular activity [13]. Its seed extract showed protective effects by decreasing liver lipid peroxides.

Leaves of Moringa oliefera (M. oliefera) contain flavanoids and flavanol glycosides, glucosinolate and isothiocyanate, phenolic acid, alkaloid and sterol important for various biological activities including bacterial activity. Thus, in the present study we prepared methanol, n-hexane, ethyl acetate and chloroform M. oliefera leaf extracts and evaluated their antibacterial activity using five pathogenic bacterial strains.

\section{Materials and methods}

Selected plant and preparation of extracts Moringa oleifera ( $M$. oliefera) leaves were collected from a home garden, Mirpur, AJK, Pakistan. Selected plant part was washed 2-3 times under running tap water and air dried for 2 weeks under shade. Later, homogenized to fine powder and stored in airtight glass bottles for extract preparation. The powdered leaf sample was soaked in $1 \mathrm{mg}: 10 \mathrm{ml}$ of methanol, n-hexane, ethyl acetate and chloroform in separate flasks for 5-7 days with 5 min shaking every day (Fig. 1). Later, filtrate was evaporated using rotary evaporator and air-dried concentrated extract was dissolved in respective solvent to prepare stock which were further diluted in broth to achieve desired concentration. 


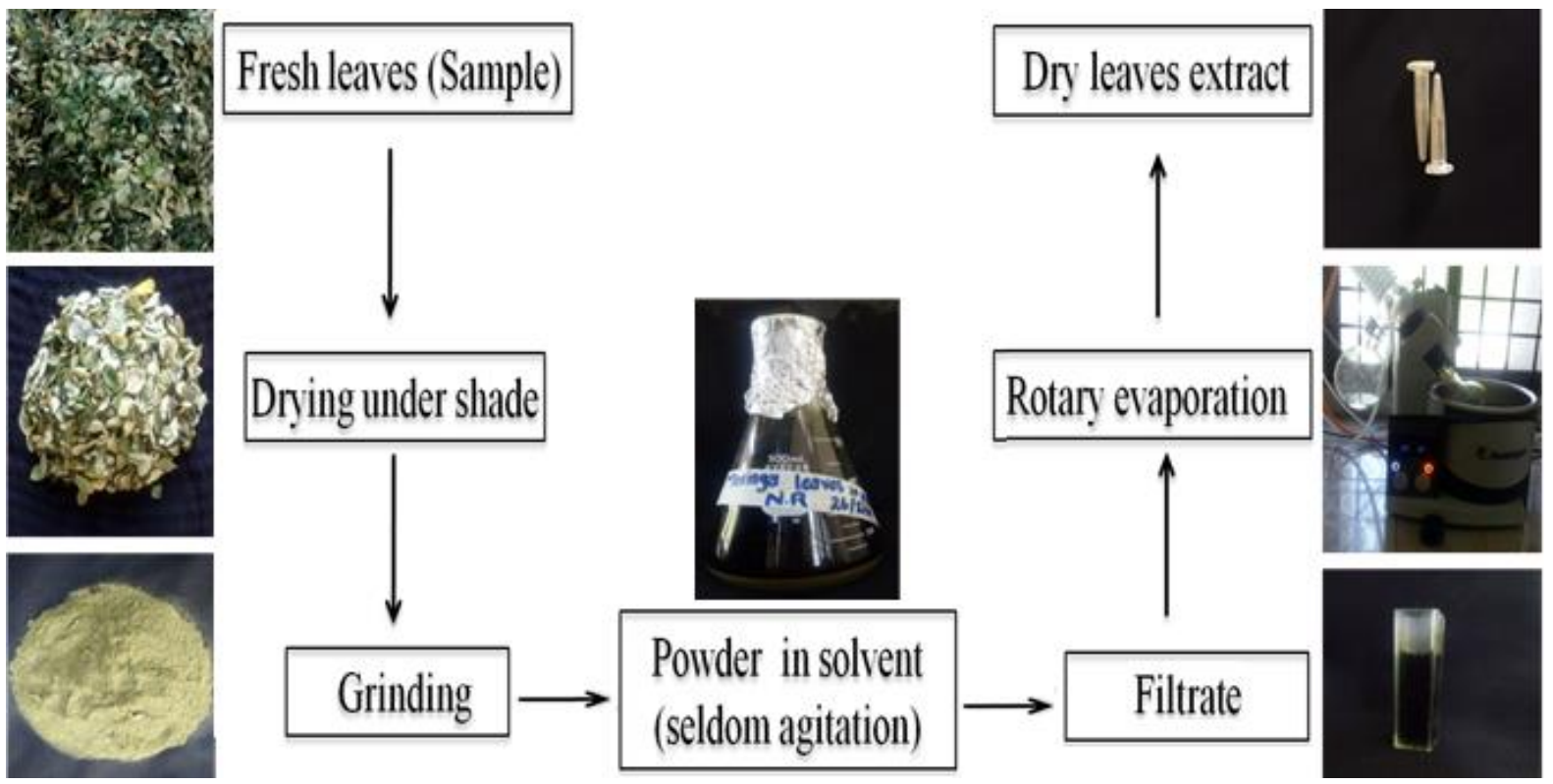

Figure 1. Different phases of Moringa oleifera leaf extracts

\section{Chemicals}

Methanol (MeOH), n-hexane (n-Hex), Chloroform $\left(\mathrm{CHCl}_{3}\right)$, Ethyl acetate (ETAC), 1, 1-di-phenyl-2-picryl hydrazyl (DPPH), analytical methanol, ethanol and ascorbic acid were used. Nutrient broth (Merck, Germany) and nutrient agar (OXOID CM003, UK) were used.

\section{Bacterial strains and culture conditions}

The bacterial strains Bacillus cereus (B. cereus) ATCC 10876, Staphylococcus aureus (S. aureus) ATCC 2592, Streptococcus pyogenes (S. pyogenes) ATCC 12384 and Salmonella enterica (S. enterica) were used in this study. Bacteria were streaked on agar plates, incubated at $37^{\circ} \mathrm{C}$ for $17 \mathrm{hrs}$. Later, plates were stored at $4{ }^{\circ} \mathrm{C}$ or used to prepare liquid culture in nutrient broth (Merck, Germany).

\section{Evaluation of antibacterial susceptibility} by well diffusion method

To evaluate the antibacterial effects of prepared extracts, well diffusion method was used [16]. Briefly, bacteria were spread homogeneously on agar plates. After short air dry, 5 wells $(6 \mathrm{~mm}$ in diameter) were made with the help of sterilized cork-borer. Then, each well was loaded with $50 \mu \mathrm{l}$ of 480 $\mathrm{mg} / \mathrm{ml}, 240 \mathrm{mg} / \mathrm{ml}, 120 \mathrm{mg} / \mathrm{ml}, 60 \mathrm{mg} / \mathrm{ml}$ and $0 \mathrm{mg} / \mathrm{ml}$ (control) of test extract. Plates were incubated at $37^{\circ} \mathrm{C}$ for $24 \mathrm{hrs}, 48 \mathrm{hrs}$ and $72 \mathrm{hrs}$, photographed and zones of inhibition around the wells were measured in $\mathrm{cm}$ as an indicator of bacterial susceptibility.

Testing bacterial inhibitory concentration $\left(\mathrm{IC}_{50}\right)$ by broth dilution method

Bacterial inhibitory concentration of test extracts was determined by macro-broth dilution method. Serial dilutions of extract were placed in sample tubes having bacteria and, control tubes having broth only (no bacteria). The total volume per tube was adjusted as $1 \mathrm{ml}$ with broth. Broth cultures were incubated at $37^{\circ} \mathrm{C}$ for $24 \mathrm{hrs}$. Later, $\mathrm{IC}_{50}$ value in $\mathrm{mg} / \mathrm{ml}$ was calculated for each extract against all test bacteria to compare effects in liquid medium.

\section{Statistical analysis}

All experiments were performed three times in triplet. Student's t-test was applied to check significant difference using lowest zone of inhibition (except zero) vs each tested zone of inhibition $\left({ }^{\mathrm{a}} \mathrm{p}<0.05 ;{ }^{\mathrm{b}} \mathrm{p}<0.005\right.$, $\left.{ }^{c} \mathrm{p}<0.0001\right)$ [17].

\section{Results and discussion}

\section{Antibacterial susceptibility}

In the present study different $M$. oliefera leaves extracts were prepared using methanol, n-hexane, chloroform and ethyl acetate. Later, their antibacterial effect against B. cereus, E. coli, S. enterica, S. aureus and $S$. pyogenes was compared in concentration dependent and incubation dependent manners. 


\section{Antibacterial effect of methanolic leaf extract}

The tested methanolic $(\mathrm{MeOH})$ leaf extract concentrations i.e. $60 \mathrm{mg} / \mathrm{ml}, 120 \mathrm{mg} / \mathrm{ml}, 240$ $\mathrm{mg} / \mathrm{ml}$ and $480 \mathrm{mg} / \mathrm{ml}$ showed $0 \mathrm{~cm}, 0 \mathrm{~cm}$, $0.09 \mathrm{~cm}, 0.15 \mathrm{~cm}$ zones of inhibition against $B$. cereus, $0 \mathrm{~cm}, 0 \mathrm{~cm}, 0.13 \mathrm{~cm}, 0.22 \mathrm{~cm}$ zones of inhibition against $S$. aureus, $0 \mathrm{~cm}, 0 \mathrm{~cm}$, $0.09 \mathrm{~cm}, 0.17 \mathrm{~cm}$ zones of inhibition against $S$. pyogenes, $0 \mathrm{~cm}, 0 \mathrm{~cm}, 0.1 \mathrm{~cm}, 0.15 \mathrm{~cm}$ zones of inhibition against $S$. enterica and $0.01 \mathrm{~cm}$, $0.1 \mathrm{~cm}, 0.18 \mathrm{~cm}, 0.3 \mathrm{~cm}$ zones of the inhibition against E. coli, respectively at $24 \mathrm{hr}$ incubation (Table 1). Thus, bacterial susceptibility trend observed at highest tested concentration $(480 \mathrm{mg} / \mathrm{ml})$ at $24 \mathrm{hr}$ incubation was as E. coli $>S$. aureus $>S$. pyogenes $>S$. enterica $>B$. cereus. Interestingly, highest zones of inhibition were observed at $24 \mathrm{hr}$ than $72 \mathrm{hr}$ incubation at all tested concentrations as shown in Table 1.

\section{Antibacterial effect of $n$-hexane leaf extract}

The tested n-hexane (n-Hex) leaf extract concentrations i.e. $60 \mathrm{mg} / \mathrm{ml}, 120 \mathrm{mg} / \mathrm{ml}, 240$ $\mathrm{mg} / \mathrm{ml}$ and $480 \mathrm{mg} / \mathrm{ml}$ showed $0.45 \mathrm{~cm}$, $0.5 \mathrm{~cm}, 0.6 \mathrm{~cm}, 0.61 \mathrm{~cm}$ zones of inhibition against $B$. cereus, $0.42 \mathrm{~cm}, 0.48 \mathrm{~cm}, 0.5 \mathrm{~cm}$, $0.57 \mathrm{~cm}$ zones of inhibition against $S$. aureus, $0.37 \mathrm{~cm}, 0.44 \mathrm{~cm}, 0.47 \mathrm{~cm}, 0.49 \mathrm{~cm}$ zones of inhibition against $S$. pyogenes, $0.32 \mathrm{~cm}$, $0.36 \mathrm{~cm}, 0.44 \mathrm{~cm}, 0.53 \mathrm{~cm}$ zones of inhibition against $S$. enterica and $0.31 \mathrm{~cm}, 0.34 \mathrm{~cm}$, $0.41 \mathrm{~cm}, 0.47 \mathrm{~cm}$ zones of the inhibition against E. coli, respectively at $24 \mathrm{hr}$ incubation (Table 1). Thus, bacterial susceptibility trend observed at highest tested concentration $(480 \mathrm{mg} / \mathrm{ml})$ at $24 \mathrm{hr}$ incubation was as B. cereus $>S$. aureus $>S$. enterica $>S$. pyogenes $>E$. coli. Interestingly, highest zones of inhibition were observed at $24 \mathrm{hr}$ than $72 \mathrm{hr}$ incubation at all tested concentrations as shown in Table 1.

\section{Antibacterial effect of ethyl acetate leaf extract}

The tested ethyl acetate (ETAC) leaf extract concentrations i.e. $60 \mathrm{mg} / \mathrm{ml}, 120 \mathrm{mg} / \mathrm{ml}$, $240 \mathrm{mg} / \mathrm{ml}$ and $480 \mathrm{mg} / \mathrm{ml}$ showed $0.28 \mathrm{~cm}$, $0.33 \mathrm{~cm}, 0.38 \mathrm{~cm}, 0.40 \mathrm{~cm}$ zones of inhibition against $B$. cereus, $0.32 \mathrm{~cm}, 0.38 \mathrm{~cm}, 0.43 \mathrm{~cm}$,
$0.47 \mathrm{~cm}$ zones of inhibition against $S$. aureus, $0.37 \mathrm{~cm}, 0.39 \mathrm{~cm}, 0.45 \mathrm{~cm}, 0.50 \mathrm{~cm}$ zones of inhibition against $S$. pyogenes, $0.27 \mathrm{~cm}$, $0.35 \mathrm{~cm}, 0.40 \mathrm{~cm}, 0.46 \mathrm{~cm}$ zones of inhibition against $S$. enterica and $0.27 \mathrm{~cm}, 0.34 \mathrm{~cm}$, $0.40 \mathrm{~cm}, 0.43 \mathrm{~cm}$ zones of the inhibition against $E$. coli, respectively at $24 \mathrm{hr}$ incubation (Table 1). Thus, bacterial susceptibility trend observed at highest tested concentration $(480 \mathrm{mg} / \mathrm{ml})$ at $24 \mathrm{hr}$ incubation was as $S$. pyogenes $>S$. aureus $>S$. enterica $>$ E. coli > B. cereus. Interestingly, highest zones of inhibition were observed at $24 \mathrm{hr}$ than $72 \mathrm{hr}$ incubation at all tested concentrations as shown in Table 1.

\section{Antibacterial effect of chloroform leaf extract}

The tested chloroform $\left(\mathrm{CHCl}_{3}\right)$ leaf extract concentrations i.e. $60 \mathrm{mg} / \mathrm{ml}, 120 \mathrm{mg} / \mathrm{ml}, 240$ $\mathrm{mg} / \mathrm{ml}$ and $480 \mathrm{mg} / \mathrm{ml}$ showed $0.19 \mathrm{~cm}$, $0.21 \mathrm{~cm}, 0.24 \mathrm{~cm}, 0.27 \mathrm{~cm}$ zones of inhibition against $B$. cereus, $0.10 \mathrm{~cm}, 0.18 \mathrm{~cm}, 0.24 \mathrm{~cm}$, $0.28 \mathrm{~cm}$ zones of inhibition against $S$. aureus, $0.15 \mathrm{~cm}, 0.21 \mathrm{~cm}, 0.26 \mathrm{~cm}, 0.29 \mathrm{~cm}$ zones of inhibition against $S$. pyogenes, $0.18 \mathrm{~cm}$, $0.26 \mathrm{~cm}, 0.31 \mathrm{~cm}, 0.38 \mathrm{~cm}$ zones of inhibition against $S$. enterica and $0.13 \mathrm{~cm}, 0.16 \mathrm{~cm}$, $0.24 \mathrm{~cm}, 0.30 \mathrm{~cm}$ zones of the inhibition against E. coli, respectively at $24 \mathrm{hr}$ incubation (Table 1). Thus, bacterial susceptibility trend observed at highest tested concentration $(480 \mathrm{mg} / \mathrm{ml})$ at $24 \mathrm{hr}$ incubation was as $S$. enterica $>E$. coli $>S$. pyogenes $>$ $S$. aureus $>B$. cereus. Interestingly, highest zones of inhibition were observed at $24 \mathrm{hr}$ than $72 \mathrm{hr}$ incubation at all tested concentrations as shown in Table1.

$M$. oliefera, chloroform root bark extract contains aglycon of deoxy-niazimicine $(\mathrm{N}$ benzyl, S-ethyl thioformate) which strongly inhibit $C$. albicans, $P$. aeruginosa, $S$. aureus, S. dysenteriae, S. boydii and S. typhi $[18,14]$. Ethyl acetate bark extract of $M$. oliefera is reported to show higher inhibition toward Staphylococcus aureus, Pseudomonas fluorescens, Citrobacter freundii and Bacillus megaterium than methanol, chloroform, and aqueous extracts of the same part of the plant [19]. 
Table 1. Measured zones of bacterial inhibition by various $M$. oliefera leaves extracts.

\begin{tabular}{|c|c|c|c|c|c|c|c|c|c|c|c|c|c|c|}
\hline \multirow{3}{*}{$\stackrel{\vec{g}}{\theta}$} & \multirow{3}{*}{  } & \multicolumn{4}{|c|}{ Concentration $(\mathrm{mg} / \mathrm{ml})$} & \multicolumn{4}{|c|}{ Concentration (mg/ml) } & \multicolumn{4}{|c|}{ Concentration (mg/ml) } & \multirow{3}{*}{ 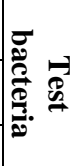 } \\
\hline & & 60 & 120 & 240 & 480 & 60 & 120 & 240 & 480 & 60 & 120 & 240 & 480 & \\
\hline & & \multicolumn{4}{|c|}{ Zone of inhibition $(\mathrm{cm})$ at $24 \mathrm{hr}$ incubation } & \multicolumn{4}{|c|}{ Zone of inhibition $(\mathrm{cm})$ at $48 \mathrm{hr}$ incubation } & \multicolumn{4}{|c|}{ Zone of inhibition $(\mathrm{cm})$ at $72 \mathrm{hr}$ incubation } & \\
\hline \multirow{20}{*}{ 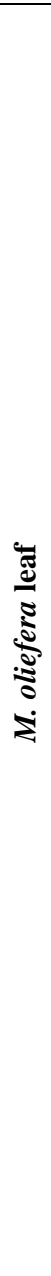 } & $\mathrm{MeOH}$ & $0.0 \pm 0.00$ & $0.0 \pm 0.00$ & $0.09 \pm 0.02$ & $0.15 \pm 0.03^{\mathrm{b}}$ & $0.00 \pm 0.00$ & $00.00 \pm 0.00$ & $0.08 \pm 0.01$ & $0.14 \pm 0.04^{b}$ & $0.00 \pm 0.00$ & $0.00 \pm 0.00$ & $0.08 \pm 0.01$ & $0.13 \pm 0.03^{\mathrm{b}}$ & \multirow{4}{*}{ 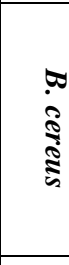 } \\
\hline & n-Hex & $0.45 \pm 0.03$ & $0.5 \pm 0.02$ & $0.60 \pm 0.03$ & $0.61 \pm 0.03$ & $0.44 \pm 0.01$ & $0.49 \pm 0.02$ & $0.56 \pm 0.02$ & $0.60 \pm 0.01$ & $0.43 \pm 0.00$ & $0.48 \pm 0.02$ & $0.55 \pm 0.03$ & $0.59 \pm 0.03$ & \\
\hline & \multirow{2}{*}{$\begin{array}{l}\text { ETAC } \\
\mathrm{CHCl}_{3}\end{array}$} & $0.28 \pm 0.01$ & $0.33 \pm 0.03$ & $0.38 \pm 0.01^{\mathrm{a}}$ & $0.40 \pm 0.02^{\mathrm{a}}$ & $0.25 \pm 0.03$ & $0.31 \pm 0.04$ & $0.38 \pm 0.02^{\mathrm{a}}$ & $0.39 \pm 0.02^{\mathrm{a}}$ & $0.22 \pm 0.02$ & $0.28 \pm 0.02$ & $0.34 \pm 0.02^{\mathrm{a}}$ & $0.37 \pm 0.02^{\mathrm{a}}$ & \\
\hline & & $0.19 \pm 0.09$ & $0.21 \pm 0.02$ & $0.24 \pm 0.03$ & $0.27 \pm 0.02^{\mathrm{a}}$ & $0.18 \pm 0.02$ & $0.20 \pm 0.02$ & $0.23 \pm 0.02$ & $0.25 \pm 0.02$ & $0.15 \pm 0.03$ & $0.17 \pm 0.02$ & $0.21 \pm 0.02$ & $0.23 \pm 0.02$ & \\
\hline & $\mathrm{MeOH}$ & $0.0 \pm 0.00$ & $0.0 \pm 0.00$ & $0.13 \pm 0.01$ & $0.22 \pm 0.02^{\mathrm{a}}$ & $0.00 \pm 0.00$ & $00.0 \pm 0.00$ & $0.12 \pm 0.02$ & $0.20 \pm 0.04^{a}$ & $0.0 \pm 0.00$ & $0.0 \pm 0.00$ & $0.11 \pm 0.02$ & $0.18 \pm 0.04^{\mathrm{a}}$ & \\
\hline & \multirow{3}{*}{$\begin{array}{l}\text { n-Hex } \\
\text { ETAC }\end{array}$} & $0.42 \pm 0.04$ & $0.48 \pm 0.03$ & $0.50 \pm 0.04$ & $0.57 \pm 0.03$ & $0.40 \pm 0.03$ & $0.46 \pm 0.03$ & $0.48 \pm 0.03$ & $0.55 \pm 0.02$ & $0.39 \pm 0.02$ & $0.45 \pm 0.02$ & $0.47 \pm 0.02$ & $0.55 \pm 0.02$ & \\
\hline & & $0.32 \pm 0.06$ & $0.38 \pm 0.03^{\mathrm{a}}$ & $0.43 \pm 0.03^{b}$ & $0.47 \pm 0.08^{b}$ & $0.29 \pm 0.03$ & $0.36 \pm 0.02^{\mathrm{a}}$ & $0.41 \pm 0.03^{b}$ & $0.46 \pm 0.02^{\mathrm{c}}$ & $0.26 \pm 0.02$ & $0.33 \pm 0.03^{b}$ & $0.40 \pm 0.02^{\mathrm{c}}$ & $0.45 \pm 0.01^{\mathrm{c}}$ & \\
\hline & & $0.1 \pm 0.04$ & $0.18 \pm 0.04^{\mathrm{a}}$ & $0.24 \pm 0.03^{b}$ & $0.28 \pm 0.02^{\mathrm{c}}$ & $0.08 \pm 0.01$ & $0.15 \pm 0.03^{\mathrm{a}}$ & $0.26 \pm 0.01^{b}$ & $0.27 \pm 0.01^{\mathrm{c}}$ & $0.09 \pm 0.01$ & $0.14 \pm 0.02^{\mathrm{a}}$ & $0.21 \pm 0.03^{b}$ & $0.25 \pm 0.00^{c}$ & \\
\hline & $\mathrm{MeOH}$ & $0.0 \pm 0.00$ & $0.00 \pm 0.00$ & $0.09 \pm$ & 0.17 & $0.00 \pm 0.00$ & 0.00 & .02 & $0.17 \pm$ & $0.00 \pm 0.00$ & $0.00 \pm 0.00$ & $0.08 \pm 0.02$ & $0.15 \pm 0.05^{\mathrm{c}}$ & \multirow{4}{*}{ 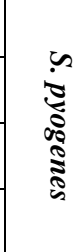 } \\
\hline & n-Hex & $0.37 \pm 0.03$ & $0.44 \pm 0.03$ & $0.47 \pm 0.04$ & $0.49 \pm 0.05^{\mathrm{a}}$ & $0.36 \pm 0.02$ & $0.42 \pm 0.02$ & $0.46 \pm 0.02$ & $0.48 \pm 0.02^{\mathrm{a}}$ & $0.33 \pm 0.06$ & $0.40 \pm 0.01$ & $0.44 \pm 0.03$ & $0.47 \pm 0.02^{\mathrm{a}}$ & \\
\hline & \multirow{2}{*}{$\begin{array}{l}\text { ETAC } \\
\mathrm{CHCl}_{3}\end{array}$} & $0.37 \pm 0.02$ & $0.39 \pm 0.02$ & $0.45 \pm 0.04^{\mathrm{a}}$ & $0.50 \pm 0.03^{\mathrm{b}}$ & $0.32 \pm 0.02$ & \pm 0.02 & $0.43 \pm 0.02^{\mathrm{a}}$ & $0.47 \pm 0.03^{b}$ & $0.29 \pm 0.02$ & 0.02 & $0.40 \pm$ & $0.44 \pm 0.04^{b}$ & \\
\hline & & $0.15 \pm 0.01$ & $0.21 \pm 0.02$ & $0.26 \pm 0.02^{\mathrm{a}}$ & $0.29 \pm 0.02^{b}$ & $0.13 \pm 0.02$ & $0.20 \pm 0.02$ & $0.23 \pm 0.03^{b}$ & $0.27 \pm 0.02^{\mathrm{b}}$ & $0.11 \pm 0.02$ & $0.18 \pm 0.04^{\mathrm{a}}$ & $0.23 \pm 0.03^{b}$ & $0.25 \pm 0.02^{\mathrm{c}}$ & \\
\hline & МeOH & $0.0 \pm 0.00$ & $0.00 \pm 0.00$ & $0.1 \pm .00$ & $0.15 \pm 0.02^{\mathrm{a}}$ & $0.00 \pm 0.00$ & $0.00 \pm 0.00$ & $0.09 \pm 0.01$ & $0.14 \pm 0.02^{\mathrm{a}}$ & $0.00 \pm 0.00$ & $0.00 \pm 0.00$ & $0.09 \pm 0.01$ & $0.14 \pm 0.02^{\mathrm{c}}$ & \multirow{4}{*}{  } \\
\hline & n-Hex & $0.32 \pm 0.00$ & $0.36 \pm 0.02$ & $0.44 \pm 0.04^{b}$ & $0.53 \pm 0.03^{c}$ & $0.3 \pm 0.00$ & $0.33 \pm 0.02$ & $0.43 \pm 0.03^{b}$ & $0.51 \pm 0.03^{c}$ & $0.29 \pm 0.01$ & $0.31 \pm 0.02$ & $0.41 \pm 0.02^{b}$ & $0.49 \pm 0.03^{c}$ & \\
\hline & \multirow{2}{*}{$\begin{array}{l}\text { ETAC } \\
\mathrm{CHCl}_{3}\end{array}$} & $0.27 \pm 0.08$ & $0.35 \pm 0.01$ & $0.40 \pm 0.03^{b}$ & $0.46 \pm 0.02^{b}$ & $0.24 \pm 0.03$ & $0.32 \pm 0.04$ & $0.38 \pm 0.02^{\mathrm{b}}$ & $0.43 \pm 0.03^{b}$ & $0.21 \pm 0.04$ & $0.30 \pm 0.04^{\mathrm{a}}$ & $0.36 \pm 0.04^{b}$ & $0.41 \pm 0.03^{c}$ & \\
\hline & & $0.18 \pm 0.00$ & $0.26 \pm 0.03$ & $0.31 \pm 0.04^{\mathrm{a}}$ & $0.38 \pm 0.03^{b}$ & $0.15 \pm 0.01$ & $0.25 \pm 0.03^{\mathrm{a}}$ & $0.29 \pm 0.04^{\mathrm{b}}$ & $0.35 \pm 0.02^{\mathrm{c}}$ & $0.13 \pm 0.04$ & $0.21 \pm 0.02^{\mathrm{a}}$ & $0.26 \pm 0.03^{b}$ & $0.34 \pm 0.02^{\mathrm{c}}$ & \\
\hline & $\mathrm{MeOH}$ & $0.01 \pm 0.00$ & $0.17 \pm 0.01$ & $0.18 \pm 0.02^{b}$ & $0.30 \pm 0.02^{\mathrm{c}}$ & $0.00 \pm 0.00$ & $0.09 \pm 0.02$ & $0.16 \pm 0.04^{\mathrm{a}}$ & $0.27 \pm 0.05^{\mathbf{c}}$ & $0.00 \pm 0.00$ & $0.15 \pm 0.10$ & $0.13 \pm 0.03$ & $0.24 \pm 0.04$ & \multirow{4}{*}{$\begin{array}{l}-1 \\
\vdots \\
\vdots\end{array}$} \\
\hline & n-Hex & $0.31 \pm 0.01$ & $0.34 \pm 0.02$ & $0.41 \pm 0.03$ & $0.47 \pm 0.03$ & $0.29 \pm 0.00$ & $0.33 \pm 0.02$ & $0.40 \pm 0.02$ & $0.46 \pm 0.02$ & $0.25 \pm 0.00$ & $0.31 \pm 0.02$ & $0.39 \pm 0.01$ & $0.45 \pm 0.02$ & \\
\hline & \multirow{2}{*}{$\begin{array}{l}\text { ETAC } \\
\mathrm{CHCl}_{3} \\
\end{array}$} & $0.27 \pm 0.01$ & $0.34 \pm 0.04^{\mathrm{a}}$ & $0.40 \pm 0.03^{b}$ & $0.43 \pm 0.02^{\mathrm{c}}$ & $0.22 \pm 0.02$ & $0.32 \pm 0.03^{c}$ & $0.37 \pm 0.04^{\mathrm{c}}$ & $0.42 \pm 0.02^{\mathrm{c}}$ & $0.19 \pm 0.04$ & $0.29 \pm 0.02^{\mathrm{c}}$ & $0.34 \pm 0.05^{\mathrm{c}}$ & $0.40 \pm 0.03^{\mathrm{c}}$ & \\
\hline & & $0.13 \pm 0.01$ & $0.16 \pm 0.08$ & $0.24 \pm 0.02^{b}$ & $0.3 \pm 0.02^{c}$ & $0.12 \pm 0.02$ & $0.15 \pm 0.08$ & $0.23 \pm 0.01^{b}$ & $0.27 \pm 0.02^{\mathrm{c}}$ & $0.09 \pm 0.01$ & $0.14 \pm 0.07$ & $0.18 \pm 0.01^{b}$ & $0.25 \pm 0.01^{\mathrm{c}}$ & \\
\hline
\end{tabular}


Rafiq et al.

Table 2. Determination of bacterial inhibitory concentration $\left(\mathrm{IC}_{50}\right)$ by broth macrodilution method

\begin{tabular}{|l|l|l|l|l|l|}
\hline \multirow{2}{*}{$\begin{array}{l}\text { Extract } \\
\text { type }\end{array}$} & \multicolumn{6}{|l}{ Bacterial inhibition IC50 \pm SEM $(\mathrm{mg} / \mathrm{ml})$} \\
\cline { 2 - 6 } & B. cereus & S. aureus & S. enterica & S. pyogenes & E. coli \\
\hline $\begin{array}{l}\mathrm{MeOH} \\
\text { extract }\end{array}$ & $9.68 \pm 0.29$ & $10.91 \pm 0.10$ & $9.02 \pm 0.00$ & $8.16 \pm 0.00$ & $7.07 \pm 0.44$ \\
\hline $\begin{array}{l}\text { n-Hex } \\
\text { extract }\end{array}$ & $1.78 \pm 0.00$ & $1.70 \pm 0.04$ & $2.11 \pm 0.11$ & $1.66 \pm 0.08$ & $1.74 \pm 0.10$ \\
\hline $\begin{array}{l}\text { ETAC } \\
\text { extract }\end{array}$ & $3.84 \pm 0.21$ & $2.58 \pm 0.13$ & $3.54 \pm 0.05$ & $3.50 \pm 0.02$ & $2.73 \pm 0.27$ \\
\hline $\begin{array}{l}\mathrm{CHCl}_{3} \\
\text { extract }\end{array}$ & $4.44 \pm 0.13$ & $4.34 \pm 0.97$ & $3.73 \pm 0.75$ & $8.36 \pm 0.20$ & $7.20 \pm 0.17$ \\
\hline
\end{tabular}

However, in the present study each leaf extract exhibited different levels of antibacterial effects depending upon test bacteria. Methanol, n-hexane, ethyl acetate and chloroform leaf extracts showed highest zone of inhibition against $E$. coli, $B$. cereus, $S$. pyogenes, $S$. aureus and $S$. enterica, respectively. Interestingly, highest zones of inhibition were observed at lowest incubation (24hr) and lowest zones were observed at $72 \mathrm{hr}$ at all tested concentrations which is in accordance to our previous findings [17].

Testing bacterial inhibitory concentration (IC50) by broth dilution method

To evaluate bacterial susceptibility in suspension state, calorimetric method was used. The extract concentration required to achieve $50 \%$ bacterial inhibition is expressed as inhibitory concentration $\left(\mathrm{IC}_{50}\right)$. Methanolic leaves extract showed $\mathrm{IC}_{50}$ values as $9.68 \mathrm{mg} / \mathrm{ml}, 10.91 \mathrm{mg} / \mathrm{ml}$, $7.07 \mathrm{mg} / \mathrm{ml}, 8.16 \mathrm{mg} / \mathrm{ml}$ and $9.02 \mathrm{mg} / \mathrm{ml}$ against $B$. cereus, $S$. aureus, E. coli, $S$. pyogenes and $S$. enterica, respectively (Table 2). The $\mathrm{IC}_{50}$ trend for methanolic leaves extract was observed as $E$. coli $>S$. pyogenes $>S$. enterica $>B$. cereus $>S$. aureus. The n-hexane leaves extract showed $\mathrm{IC}_{50}$ values as $1.78 \mathrm{mg} / \mathrm{ml}, 1.70$ $\mathrm{mg} / \mathrm{ml}, 1.74 \mathrm{mg} / \mathrm{ml}, 1.66 \mathrm{mg} / \mathrm{ml}$ and 2.11 $\mathrm{mg} / \mathrm{ml}$ against $B$. cereus, $S$. aureus, E. coli, $S$. pyogenes and $S$. enterica, respectively. The $\mathrm{IC}_{50}$ trend of n-hexane extract was $S$. pyogenes $>S$. aureus $>$ E. coli $>$ B. cereus
$>S$, enterica. Ethyl acetate leaves extract showed $\mathrm{IC}_{50}$ values as $3.84 \mathrm{mg} / \mathrm{ml}, 2.58$ $\mathrm{mg} / \mathrm{ml}, 2.73 \mathrm{mg} / \mathrm{ml}, 3.50 \mathrm{mg} / \mathrm{ml}$ and 3.54 $\mathrm{mg} / \mathrm{ml}$ against $B$. cereus, $S$. aureus, E. coli, $S$. pyogenes and $S$. enterica, respectively. The $\mathrm{IC}_{50}$ trend of ethyl acetate extract was $S$. aureus > E. coli > S. pyogenes > S. enterica > B. cereus. Moreover, chloroform leaves extract showed $\mathrm{IC}_{50}$ values as $4.44 \mathrm{mg} / \mathrm{ml}, 4.34 \mathrm{mg} / \mathrm{ml}, 7.20$ $\mathrm{mg} / \mathrm{ml}, 8.36 \mathrm{mg} / \mathrm{ml}$ and $3.73 \mathrm{mg} / \mathrm{ml}$ against B. cereus, S. aureus, E. coli, S. pyogenes and $S$. enterica, respectively. The $\mathrm{IC}_{50}$ trend of chloroform leaves was $S$. enterica $>S$. aureus $>$ B. cereus $>$ E. coli $>S$. pyogenes (Table 2). Kaur et al [20] reported that a methanolic leaves extract and $70 \%$ ethanolic extract of $M$. oliefera showed antileishmanial activity against $\mathrm{L}$. donovani promastigotes. The ethyl acetate fraction of a methanolic extract was reported to show leishmaniasis with an $\mathrm{IC}_{50}$ of $27.5 \mu \mathrm{g} / \mathrm{ml}$. Moreover, $M$. oliefera extracts are reported to exhibit antiviral, antifungal, antihyperglycemic, antihyperlipidemic, and hypocholesterolemic activities. However, in the present study methanol, nhexane, ethyl acetate and chloroform leaf extracts showed antibacterial effect against all five tested bacteria. Recently, bacteria including B. cereus, S. aureus, S. pyogenes, $S$. enterica and E. coli are reported to show resistance to commonly used antibiotics at alarming rate [1-7]. However, none of the tested bacteria showed resistance against any of the tested extract in well diffusion or 
macrodilution method expressing the $M$. oliefera leaves extracts as potent candidates to kill bacteria in semisolid or in liquid medium for bacterial inhibition.

\section{Conclusion}

In the present study four extracts from $M$. oliefera leaves were prepared using methanol, n-hexane, chloroform and ethyl acetate. All extracts expressed bacterial inhibition against all tested bacteria i.e. $B$. cereus, E. coli, S. enterica, S. aureus and $S$. pyogenes in both well diffusion (semi solid medium) and macrodilution method (liquid medium) presenting the $M$. oliefera leaves extracts as potent candidates to treat tested bacteria in future.

\section{Authors' contributions}

Conceived and designed the experiments: A Ali \& N Rafiq, Performed the experiments: N Rafiq, Analyzed the data: $\mathrm{H}$ Khurshid, B Akbar, Z H Tarar, Contributed materials/ analysis/ tools: I Ahmed, F Nazir \& M Ahmed, Wrote the paper: A Ali, N Rafiq \& H Javed.

\section{Acknowledgement}

We are thankful to MUST for financial support and NARC for providing bacteria.

The authors declare no financial or any other conflict of interest.

\section{References}

1. Mahalingam $\mathrm{R}$, Bharathidasan $\mathrm{R}$, Ambikapathy V \& Panneerselvam A (2011). Studies on antibacterial activity of some medicinal plant against human pathogenic microorganism. Asia J Plant Sci Res 1(3): 86-90.

2. Preethi R, Devanathan VV \& Loganathan M (2010). Antimicrobial and antioxidant efficacy of some medicinal plants against food borne pathogens. Adv Biol Res 4(2): 122-5.

3. Rodríguez-Bano J, Navarro MD, Romero L, Martínez-Martínez L, Muniain MA, Perea EJ, Pérez-Cano R $\&$ Pascual A (2004). Epidemiology and clinical features of infections caused by extended-spectrum beta-lactamaseproducing Escherichia coli in nonhospitalized patients. $J$ Clin Microbiol 42(3): 1089-94.

4. Y1lmaz EŞ \&Aslantaş Ö (2017). Antimicrobial resistance and underlying mechanisms in Staphylococcus aureus isolates. Asian Pac J Trop Med 10(11): 1059-1064.

5. Bottone EJ (2010). Bacillus cereus, a volatile human pathogen. Clin Microbiol Rev 23(2): 382-98.

6. Afolami OI \& Onifade AK (2018). Antibiotic Resistant Salmonella spp: Mechanism of Drug Resistance, Gene Variations and Clinical Implications. Asian J Res Med Pharma Sci 4(4): 1-6.

7. Ali NA, Jülich WD, Kusnick C \&Lindequist U (2001). Screening of Yemeni medicinal plants for antibacterial and cytotoxic activities. $J$ Ethnopharmacol 74(2): 173-179.

8. Kalaycioglu A \&Öner C (1994). Bazi bitki

ekstraksiyonlarýnýnantimutajeniketkil erinin Amest Salmonella test sistemiile Araptýrýlmasý. Turk J Bot 18: 117122.

9. Erdogrul ÖT (2002). Antibacterial activities of some plant extracts used in folk medicine. Pharm Biol 40(4): 26973.

10. Vonderbank H (1949). Results of chemotherapy for tuberculosis. Pharm 4: 198-207.

11. Ramachandran C, Peter KV \& Gopalakrishnan PK (1980). Drumstick (Moringa oleifera): a multipurpose Indian vegetable. Econ Bot 3(34): 27683.

12. Morton JF (1991). The horseradish tree, Moringa pterygosperma (Moringaceae)-a boon to arid lands. Economic Bot 45(3): 318-33.

13. Siddhuraju P \& Becker K (2003). Antioxidant properties of various solvent extracts of total phenolic constituents from three different agro climatic origins of drumstick tree (Moringa oleifera Lam.) leaves. $J$ Agric Food Chem 51(8): 2144-2155. 
14. Rani A, Zahirah N, Husain K \& Kumolosasi E (2018). Moringa genus: a review of phytochemistry and pharmacology. Front Pharmacol 9(108): 1-26.

15. Dillard CJ \& German JB (2000). Phytochemicals: nutraceuticals and human health. J Sci Food Agric 80(12): 1744-1756.

16. Albuquerque CC, Camara TR, Mariano RD, Willadino L, Marcelino Júnior C \&Ulisses C (2006). Antimicrobial action of the essential oil of Lippiagracilis Schauer. Braz Arch Biol Technol 49(4): 527-535.

17. Khurshid H, Rafiq M, Nazir F, Ali I, Ahmed M, Akbar B, Ahmed M \& Ali A (2019). Antimicrobial properties of hydrogen peroxide and potash alum alone and in combination against clinical bacterial isolates. Pure Appl Biol 8(4): 2238-2247.

18. Nikkon F, Saud ZA, Rahman MH \& Haque ME (2003). In vitro antimicrobial activity of the compound isolated from chloroform extract of Moringa oleifera Lam. PakJ Biol Sci 6(22): 1888-1890.

19. Zaffer M, Ahmad S, Sharma R, Mahajan S, Gupta A \& Agnihotri RK (2014). Antibacterial activity of bark extracts of Moringa oleifera Lam. against some selected bacteria. Pak J Pharm Sci 27(6):1857-1862.

20. Kaur A, Kaur PK, Singh S \& Singh IP (2014).

Antileishmanial compounds from Moringa oleifera Lam. Z Naturforsch C J Biosci 69(3-4): 110-116. 